\title{
Retraction Note to: Field-Induced Superconductivity in $\mathrm{MoS}_{2}$
}

\author{
J. T. Ye $\cdot$ Y. J. Zhang $\cdot$ M. Yoshida $\cdot$ Y. Saito $\cdot$ Y. Iwasa
}

Published online: 5 September 2014

(C) Springer Science+Business Media New York 2014

Retraction to: J Supercond Nov Magn (2014)

\section{7:981-985}

DOI 10.1007/s10948-013-2422-y

Because of excessive overlap with Ye, J.T., Zhang, Y.J., Akashi, R., Bahramy, M.S., Arita, R., Iwasa, Y.: Superconducting dome in a gate-tuned band insulator. Science 338, 1193 (2012). doi: 10.1126/science.1228006, Ye, J.T., Zhang, Y.J., Yoshida, M., Saito, Y., Iwasa, Y.: Field-induced superconductivity in $\mathrm{MoS}_{2}$. Journal of Superconductivity and Novel Magnetism 27, 981-985 (2014). doi: 10.1007/s10948-013-2422-y has been withdrawn at the request of the authors.

The online version of the original article can be found at http:// dx.doi.org/10.1007/s10948-013-2422-y.

J. T. Ye · Y. J. Zhang · M. Yoshida · Y. Saito · Y. Iwasa

Quantum-Phase Electronics Center and Department of Applied

Physics, The University of Tokyo, 7-3-1 Hongo, Bunkyo,

Tokyo 113-8656, Japan

J. T. Ye $(\bowtie)$

Device Physics of Complex Materials, Zernike Institute

for Advanced Materials, University of Groningen, Nijenborgh 4,

9700 AE Groningen, The Netherlands

e-mail: j.ye@rug.nl

Y. Iwasa

CERG, RIKEN, Hirosawa 2-1, Wako 351-0198, Japan 